\title{
Simulation on the effective guidance model for data flow in large scale multimedia teaching system
}

\author{
Liuwei \\ Yunyang Teachers'College, Hubei Shiyan 442000, China
}

Keywords: multimedia; teaching system; data flow guidance;

\begin{abstract}
Effective guidance on data flow in large multimedia teaching system, can service student to the greatest degree. With the growth of covered data of multimedia teaching system, a lot of useful teaching information will be drown in the deluge of information in multimedia teaching. Using the traditional method for data flow guiding in large scale multimedia teaching system, it will be disturbed by the mass information, making the time-consuming data flow guide process, and low efficiency. Therefore, an effective guidance method for data flow in large-scale multimedia teaching system based on fuzzy support vector machine algorithm is proposed. Data attributive character of large multimedia teaching system is extracted, to provide accurate data base for data flow guidance. According to the theory of fuzzy support vector machine, obtain the optimal classification plane, so as to realize the optimization process of data flow guidance in the large multimedia teaching system. The experimental results show that using the improved algorithm for data flow guidance in the large multimedia teaching system, and can effectively shorten the data flow guiding time and ensure the results of data flow guiding to meet the requirements of teaching.
\end{abstract}

\section{Introduction}

With the rapid increase of data flow guiding technology, data flow guidance technology has been widely used in data management of various industries, playing a more and more important role [1]. In the field of education, with the rapid increase varieties and the number of teaching information, a lot of useful information will be submerged in the vast amounts of information[2], therefore, effectively guiding on the data flow, has become the core problem to research in the field of data management [3]. The data flow guiding technology applied in large scale multimedia teaching system, can enhance the data management ability of multimedia teaching system. Therefore, large multimedia teaching system's data flow guiding method, become the key subject to study in the field of data [4]. At present stage, the main methods for data stream guidance in large-scale multimedia teaching system, include the method based on the improved support vector machine algorithm [5], Gauss model [6] and fuzzy clustering algorithm [7]. Among them, the most commonly used is the method for data stream guidance based on improved support vector machine algorithm in large multimedia teaching system [8]. Because the data flow guiding methods play an irreplaceable role in the field of information management, it has a broad development prospect, becoming the focus of many experts study [9].

With the growth of the data covered in multimedia teaching system, a lot of useful teaching information will be drown in the deluge of information in multimedia teaching. Using the traditional method for data flow guiding in large scale multimedia teaching system, it will be disturbed by the mass information, making the time-consuming process of data flow guidance, and low efficiency [10].

In order to avoid the disadvantages of the traditional algorithm, an effective guidance method for data flow in large-scale multimedia teaching system based on fuzzy support vector machine algorithm is proposed. Data attributive character of large multimedia teaching system is extracted, to provide accurate data base for data flow guidance. According to the theory of fuzzy support vector machine, obtain the optimal classification plane, so as to realize the optimization process of data flow guidance in the large multimedia teaching system. The experimental results show that using the improved algorithm for data flow guidance in the large multimedia teaching system, and can effectively shorten the data flow guiding time and ensure the results of data flow guiding to 
meet the requirements of teaching.

\section{The principle of large multimedia teaching system's data flow guiding}

According to the related principle of improved support vector machine, it can effectively guide the data flow in large multimedia teaching system, its principle is as follows:

Setting that the composed set by teaching information data in large-scale multimedia teaching system is $\left\{\left(y_{j}, z_{j}\right)\right\}$, including $j=1,2, \cdots, m, y_{j} \in V^{r}, Z,{ }_{j} \in\{1,2, \cdots, Q\}, m$ is the number of all the data in teaching information, $Q$ is the number of teaching information data attribute types, $V^{r}$ is a storage location in data $r$ dimensional space. According to the improved support vector machine algorithm, $V^{r}$ can be divided into $Q$ different categories. In the teaching system, the same teaching information data have many different attributes, use the following formula can make the classification of multi characteristics:

$$
\begin{aligned}
& \min _{f_{1}, d_{1}, \alpha_{1}} \frac{1}{2}\left\|f_{j}\right\|^{2}+E \sum_{j=1}^{l} \alpha_{j, k} \\
& f_{j} \cdot \mu\left(y_{k}\right)+d_{j} \geq 1-\alpha, \text { if } z_{j}=j \\
& f_{j} \cdot \mu\left(y_{k}\right)+d_{j}<-1+\alpha_{k, l} \text { if } z_{j} \neq j
\end{aligned}
$$

Using the following formula describe the function of teaching data attribute analysis:

$$
\left\{\begin{array}{l}
h_{1}(y)=\operatorname{ali}\left(f_{1} \cdot y+v_{1}\right) \\
\vdots \\
h_{R}(y)=\operatorname{ali}\left(f_{R} \cdot y+v_{R}\right)
\end{array}\right.
$$

The above formula is calculate to get the multimedia teaching data $y$. Using the above analysis function, it can carry on the effective analysis of the teaching information data. The formula is as follows:

$$
\operatorname{Team}(y)=\arg \max _{j=1,2, \cdots, Q}\left(x_{j} \cdot y+d_{j}\right)
$$

According to the above method, it can be obtained the data flow guiding function of multimedia teaching information $\frac{Q(Q-1)}{2}$, the formula is as follows:

$$
h_{j k}(y)=\operatorname{alk}\left(f^{j k} \cdot y+e^{j k}\right)
$$

in the above formula, $k=1,2, \cdots, Q$ and $j \neq k$ 。

In the process of large multimedia teaching system's data flow guidance, due to the same kind of multimedia teaching information attribute has diversity, therefore, it needs to reduce redundant data characteristics. The detailed method are described below:

Using the following formula calculate the similarity coefficient of data in multimedia teaching system:

$$
\operatorname{sim}\left(w_{b}, w_{c}\right)=\frac{\sum_{l=1}^{n} X_{b l} \times \sum_{l=1}^{n} X_{c l}}{\sqrt{\sum_{l=1}^{n} X_{b l}^{2} \times \sum_{l=1}^{n} X_{c l}^{2}}}
$$

Using the following formula build large multimedia teaching system's data flow guide model, to effectively guide the data flow:

$$
Y^{\prime}=(Y-\bar{Y}) / T, T=\sqrt{\frac{\sum_{j=1}^{p}\left(Y_{j}-\bar{Y}\right)^{2}}{p-1}}
$$

According to the method described above, it can classify large multimedia teaching information, the teaching information in accordance with the data characteristics of the teaching information are will be divided into several categories, to provide accurate data base for large multimedia teaching system's data flow guidance. According to different categories of teaching information data, data flow guidance is perpormed. 


\section{Method for data flow guiding in large multimedia teaching system}

\section{Extraction of teaching data attributes}

By using the method of local characteristics analysis, extract the key features of the teaching data in large multimedia teaching system, and make the analysis of these data, to be as the data flow guidance foundation. This characteristic method is not affected by the delay variation in the process of data acquisition, through the comparison of key characteristics of teaching data in large multimedia teaching system and data characteristics values in the adjacent area, the feature of the teaching data can be described.

The similarity of teaching data can be set used $E_{s}^{\min }$ to describe, then we can get the following formula:

$$
\begin{aligned}
& E_{S}^{\min }(M)=\min \left\{E_{S}\left(M, M^{\text {uniform }}\right), \text { all } M^{\text {uniform }}\right\} \\
& E_{S}\left(M, M^{\text {uniform }}\right)=\min \left\{\varphi\left(S\left(M_{i}\right), M^{\text {uniform }}\right\}\right.
\end{aligned}
$$

\section{Description of data flow guidance process}

According to the relevant principles of optimal classification plane, the data of multimedia teaching can be guided. The data flow guidance problem is transformed into an optimal plane solving, and using the following formula to describe the problem to be solved:

$$
R(\beta)=\sum_{j=1}^{p} \beta_{j}-\frac{1}{2} \sum_{j, k=1}^{p} \beta_{j} \beta_{k} z_{j} z_{k}\left(y_{j} \cdot y_{k}\right)
$$

The above mentioned problem of optimal classification plane solving need to satisfy the following constraints:

$$
\left\{\begin{array}{l}
\sum_{j=1}^{p} z_{j} \beta_{j}=0 \\
\beta_{j} \geq 0, j=1,2, \cdots, p
\end{array}\right.
$$

Assuming that the characteristics of multimedia teaching data appears nonlinear transformation, it needs to use the inner product $L\left(y, y^{T}\right)$ to alternate the dot product of optimal classification function. The results of optimal classification plane solving problem after transformation can use the following formula to describe:

$$
R(\beta)=\sum_{j=1}^{p} \beta_{j}-\frac{1}{2} \sum_{j, k=1}^{p} \beta_{j} \beta_{k} z_{j} z_{k} L\left(y_{j} \cdot y_{k}\right)
$$

The optimal classification functions corresponding to the above formula can be described by using the following formula:

$$
g(y)=\operatorname{sgn}\left(\sum_{j=1}^{p} \beta_{j} z_{j} L\left(y_{j}, y_{k}\right)+c^{\prime}\right)
$$

The optimal classification plane method can classify the two different types of teaching data, and the data flow guiding question of large multimedia teaching system is a multi-category classification problem. Therefore, the data flow guide problem in large multimedia teaching system is needed to be into multiple of two classification problems, and solve the above problems respectively, so as to obtain the results of data flow guidance in the large multimedia teaching system.

\section{Experiment results and analysis}

In order to verify the effectiveness of improved algorithms, it needs an experiment, the experimental environment is Visual $\mathrm{C}++6.0$.

When the sample complexity is low, the obtained results of respectively using the traditional algorithm and the improved algorithm for data flow guidance in large multimedia teaching system, can be described by the following table 1 : 
Table 1 experimental results of when sample complexity is low

\begin{tabular}{|c|c|c|}
\hline $\begin{array}{l}\text { The } \\
\text { number } \\
\text { of } \\
\text { experim } \\
\text { ents }\end{array}$ & $\begin{array}{l}\text { Time-consuming of } \\
\text { traditional algorithm } \\
(\mathrm{ms})\end{array}$ & $\begin{array}{l}\text { Time-consuming of } \\
\text { improved algorithm } \\
(\mathrm{ms})\end{array}$ \\
\hline 1 & 12 & 11 \\
\hline 2 & 14 & 12 \\
\hline 3 & 12 & 11 \\
\hline 4 & 13 & 13 \\
\hline 5 & 15 & 12 \\
\hline 6 & 16 & 11 \\
\hline 7 & 13 & 14 \\
\hline 8 & 15 & 11 \\
\hline 9 & 13 & 12 \\
\hline 10 & 13 & 14 \\
\hline
\end{tabular}

When the sample complexity is high, obtaining results of respectively using the traditional algorithm and the improved algorithm for data flow guidance in large multimedia teaching system, can be described by the following table:

\begin{tabular}{|c|c|c|}
\hline $\begin{array}{l}\text { The } \\
\text { number } \\
\text { of } \\
\text { experim } \\
\text { ents }\end{array}$ & $\begin{array}{l}\text { Time-consuming of } \\
\text { traditional algorithm } \\
\text { (ms) }\end{array}$ & $\begin{array}{l}\text { Time-consuming of } \\
\text { improved algorithm } \\
(\mathrm{ms})\end{array}$ \\
\hline 1 & 21 & 14 \\
\hline 2 & 23 & 12 \\
\hline 3 & 22 & 12 \\
\hline 4 & 23 & 15 \\
\hline 5 & 25 & 12 \\
\hline 6 & 22 & 14 \\
\hline 7 & 26 & 16 \\
\hline 8 & 23 & 13 \\
\hline 9 & 21 & 16 \\
\hline 10 & 24 & 17 \\
\hline
\end{tabular}

According to the experiment on the table, it can be learned that, when the sample complexity is low, the consuming time of the use of traditional algorithm and the improved algorithm for data flow guiding is basically the same, but, when the sample complexity is high, using the improved algorithm to guide the data flow in large multimedia teaching system can improve the accuracy of data flow guidance.

The above experimental data table, will be able to get below: 


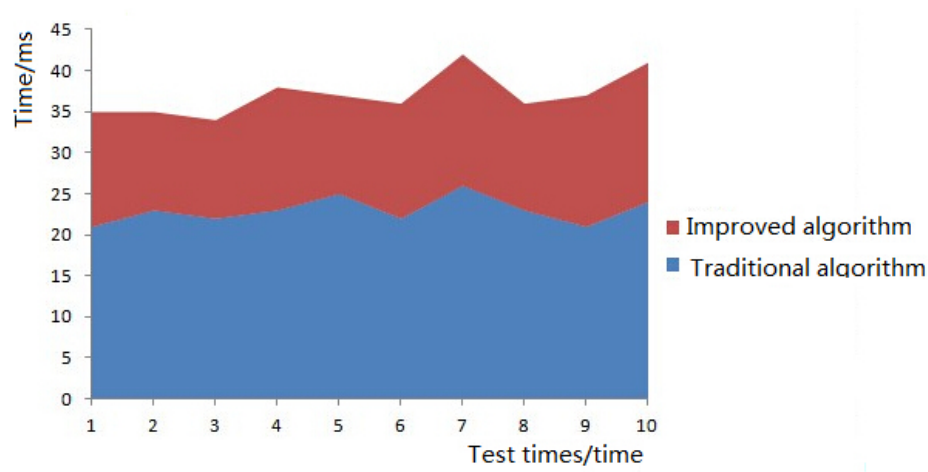

Figure 1 time consuming comparing different algorithms

According to the above to know, in the case of sample complexity is higher, the improved algorithm, shorter than the traditional algorithm, the amount of time fully shows the superiority of the algorithm in this paper.

\section{Conclusion}

An effective guidance method for data flow in large-scale multimedia teaching system based on fuzzy support vector machine algorithm is proposed in this paper. Data attributive character of large multimedia teaching system is extracted, to provide accurate data base for data flow guidance. According to the theory of fuzzy support vector machine, obtain the optimal classification plane, so as to realize the optimization process of data flow guidance in the large multimedia teaching system. The experimental results show that using the improved algorithm for data flow guidance in the large multimedia teaching system, and can effectively shorten the data flow guiding time and ensure the results of data flow guiding to meet the requirements of teaching.

\section{References}

[1] Guo Luodan, Kong Jinsheng. Application of MRBF neural network in image classification [J]. Computer engineering and design, 2009, 30 (13): 3154-3156.

[2] Deng Huawu, Clausi D A. Gaussian MRF rotation-invariant features for image classification [J]. IEEE Transactions on Pattern Analysis and Machine Intelligence, 2004, 26(7): 162-165.

[3] Zhang Jianping, Liu Xiya. Research and application of K means algorithm based on clustering analysis [J]. Computer research and application, 2007, 24 (5): 166-168.

[4] Xie Wenlan, Shi Yuexiang, Xiao Ping. Application of BP neural network in the natural image classification [J]. Computer engineering and application, 2010, 46 (2): 162-166.

[5] Zhang Jinshui, He Chunyang, Pan Yaozhong, et al. Study on classification of high spatial resolution remote sensing data with multi-source information composition based on SVM [J]. Remote sensing journal, 2006, 10 (1): 49-57.

[6] Niu Peng, Wei Wei. Hyperspectral remote sensing image classification based on SVM dynamic integration [J]. Computer applications, 2010, 30 (6): 1590-1593.

[7] Ouyang Sen, Song Zhengxiang, Wang Jianhua, et al. The power quality denoising algorithm based on signal correlation and wavelet method [J]. Transactions of China Electrotechnical Society, 2003, 18 (3): 112-116.

[8] Sun Yizhong. The theory and application basic of XML. Beijing: Beijing University of Posts and Telecommunications press, 2000:130-134

[9] Wan Changxuan, Liu Xiping. XML database technology. Beijing: Tsinghua University press.2008:137-200 
[10] Hu Zhaoling, Li Haiquan. Research of SAR image texture feature extraction and classification [J]. Journal of China University of Mining and Technology, 2009, 38 (3): 422-427.

[11] Kuklik P, Szumowski L, Zebrowski J J, et al. Integration of the data from electroanatomical mapping system and CT imaging modality[J]. International Journal of Cardiovascular Imaging. 2009, 25(4): 425-432. 